\title{
Adapting IT Algorithms and Protocols to an Intelligent Urban Traffic Control
}

\author{
Levente Alekszejenkó $^{1}$ and Tadeusz Dobrowiecki ${ }^{2}$
}

\begin{abstract}
Autonomous vehicles, communicating with each other and with the urban infrastructure as well, open opportunity to introduce new, complex and effective behaviours to the intelligent traffic systems. Such systems can be perceived quite naturally as hierarchically built intelligent multi-agent systems, with the decision making based upon well-defined and profoundly tested mathematical algorithms, borrowed e.g. from the field of information technology.

In this article, two examples of how to adapt such algorithms to the intelligent urban traffic are presented. Since the optimal and fair timing of the traffic lights is crucial in the traffic control, we show how a simple Round-Robin scheduler and Minimal Destination Distance First scheduling (adaptation of the theoretically optimal Shortest Job First scheduler) were implemented and tested for traffic light control. Another example is the mitigation of the congested traffic using the analogy of the Explicit Congestion Notification ( $E C N$ ) protocol of the computer networks. We show that the optimal scheduling based traffic light control can handle roughly the same complexity of the traffic as the traditional light programs in the nominal case. However, in extraordinary and especially fastly evolving situations, the intelligent solutions can clearly outperform the traditional ones. The ECN based method can successfully limit the traffic flowing through bounded areas. That way the number of passing-through vehicles in e.g. residential areas may be reduced, making them more comfortable congestion-free zones in a city.
\end{abstract}

Index Terms-intelligent traffic control, connected vehicles, congestion notification, Intelligent Transportation Systems (ITS), Intelligent Traffic Light System (ITLS)

\section{INTRODUCTION}

$\mathbf{A}$ $\mathrm{S}$ our vehicles become more and more sophisticated (up to being self-driving and autonomous, smart cars for convenience) and the traffic infrastructure itself also evolves, communication between smart cars $(\mathrm{V} 2 \mathrm{~V})$, or between smart cars and various parts of the infrastructure (V2I), or even between various elements of the infrastructure (intersections, parking lots, etc.) is no longer a fiction. If the infrastructure and the smart cars are also capable of cooperative actions by following the exchanged communication messages, it is possible to form intelligent multi-agent systems to improve road safety, reduce traveling times, costs and pollution, or even to mitigate congestion as well. For more details, see Section III.

However, the internal behavior (the decision making) of these agents has to be defined. Among others such agents have

\footnotetext{
1,2 Balatonfüred Student Research Group

${ }^{2}$ Budapest University of Technology and Economics, Budapest, Hungary (e-mail: ale.levente@gmail.com and tade@mit.bme.hu)
}

to calculate answers to the e.g. following questions: Would it be beneficial for a smart car to join a group of cars in front of it? When should an intelligent traffic light provide a green-light for a particular platoon of smart cars? When shall an intelligent traffic light ask one of its neighbor junctions to reduce its output to prevent congestion? To be able to answer these questions, Round-Robin, Minimal Destination Distance First, and Explicit Congestion Notification protocols are proposed in Section IV. When we defined these methods, we had the presumption that every vehicle in the traffic are autonomous and can communicate with each other.

Besides integrating various components of an intelligent transportation system into a hierarchical multi-agent system, adopting the aforementioned protocols to the road traffic domain, especially the ECN protocol, is the principal novelty in our research. The proposed solutions were also tested by simulations of different (hopefully realistic) scenarios, using the Eclipse SUMO microscopic traffic simulator tool [1]. The measurements and their results are summarized in Section V.

\section{LITERATURE REVIEW}

As the first coordinated traffic lights were created more than one hundred years ago [2], the literature of traffic control contains many interesting articles, books, and lecture-notes. Even though this is a well-researched area, perhaps the major problem of transportation, the congestion, still exists.

Traffic signal coordination, green-waves, are nowadays mainly created by methods depending on analyzing statistical data, like TRANSYT and SCOOT [3]. Since those algorithms were created decades ago, they might not be able to handle the problems of today's traffic. Thus, it may be helpful to implement new, intelligent methods into the traffic lights. One of these approaches may be the usage of Minimal Destination Distance First [4] control which is analogous to the theoretically optimal scheduling algorithm, called the shortest job first. Unfortunately, this method is unfair on its own, therefore it shall be modified to use it in real-life [5].

It is natural to treat the participants of urban traffic (e.g. vehicles, infrastructural elements, traffic lights, etc.) as a multiagent system. In this framework, novel ideas can also be experimented with, such as a time-slot booking to pass through at the intersections, explained in [6]. Unfortunately, there is no guarantee that a smart vehicle will arrive on-time to a certain intersection, but this method contains the possibility to withdraw the already posted bookings. The problem is that the state-space of such a system can be enormous, therefore this 
and similar algorithms require a vast amount of computational time and memory space.

Method of significantly lower complexity is proposed in [7]. This proposal varies the phase time of traffic lights like the SCOOT method does, but this method varies the phase times of multiple traffic lights at the same time. Therefore it creates arterial directions. As our research showed [5], the main advantage of intelligent traffic control is that it behaves better in extraordinary situations. In the investigated cases, particular arterial directions were closed, and secondary routes opened to obligatory use, due to the road closures. Therefore in our research, we tried to avoid creating arterial directions.

In a grid-like road network, for example, typical to the U.S., there are at least two routes with the same cost between any two points of the road network. [8] takes advantage of this fact, optimizing traffic both in time and space in over-saturated scenarios. Unfortunately, this method cannot be simply applied in irregularly shaped road networks, prevalent e.g. in Europe.

Traffic flow can also be described with the concepts borrowed from Economics. Therefore some economical formulas and methods also can be applied in the domain of road traffic. [9] presents an economical approach to optimize the flow of traffic. However, it is not a true real-time solution, since the phases shall be recalculated always when a new car approaches an intersection. Therefore this method is also really of high computational complexity.

Computationally, a much simpler approach is to create individual agents at traffic lights and design an algorithm or a physical phenomenon which automatically provides the signal coordination. [10] shows that traffic coordination might easily be implemented by actuated traffic lights. In this case, communication between traffic light is not necessary, since the incoming platoons of vehicles can synchronize those intersection managers when they arrive at the corresponding induction loop detectors. [11] also suggest using a distributed traffic light control, in which controller agents can play an evolutionary game. By playing the game individually, the agents might be able to find the globally optimal solution as well. Unfortunately, this work does not mention what happens when the system is adapting to the recently changed traffic. There is a possibility that an almost endless traffic congestion forms in this transient state (considering that both the traffic itself and the traffic controllers are in a transient state for a while).

For this reason, our ECN-based solution, presented in this paper, is a much more conservative one. It is also a distributed solution, but a limited amount of information is shared among the topologically neighboring intelligent traffic controllers. Based on this information, our method solves a relatively small optimization (integer programming) problem. [12] attests that sharing information with neighboring intersection managers can be beneficial for targeting the globally optimal solution. Based on these results, optimizing the scheduling of more intersection controllers (e.g. for a dedicated direction, [13]) at the same time might not be worth the increased computational time and complexity.

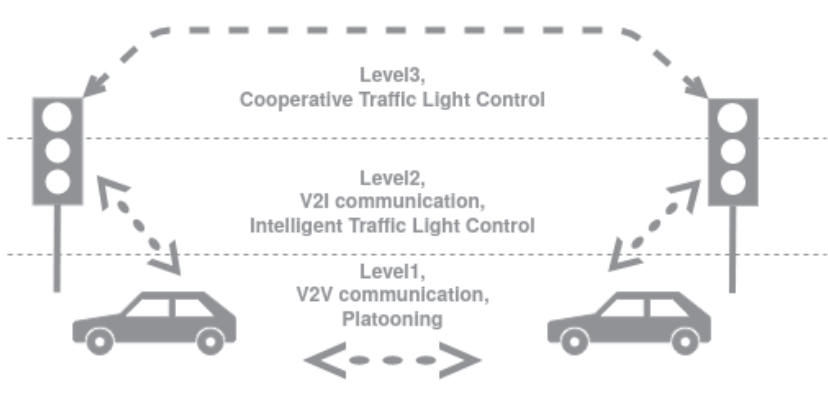

Fig. 1. Example of a three-layered, intelligent multi-agent system of urban traffic.

\section{URban TrafFic as AN Intelligent Multi-AGENT SYSTEM}

Autonomous vehicles, smart traffic lights are intelligent agents on their own. By using their capability of communication, cooperative, multi-agent systems can be formed. ${ }^{3}$ In the following, we assume these agents to be trustworthy and bonafide, cooperative, and being able to perform actions prescribed by the defined protocols. The communication itself is free of lost packets, the bandwidth is enough to transmit all the messages and the delay of the transmission does not have any effect on the agents' behavior. On this basis, we can identify three layers of the cooperating agents and the related intelligent behavior.

In the first layer (lowest, vehicle-level, see Figure 1), intervehicular communication is used to form groups of smart cars, the so-called platoons. Vehicles in a platoon can keep shorter following distances and can perform some maneuvers together, like e.g. changing lanes. When multiple vehicles change lanes together, they might have a smaller impact on the flow of the traffic, compared to changing lanes individually. Unfortunately, in an urban environment, there is usually not enough space and time to perform complex maneuvers, therefore we believe only simple methods can be executed there. Thus, platoons in urban scenarios are expected to form in ad-hoc ways, in smaller groups, and will have a relatively shorter lifetime.

Besides using simple platoon movements, there are many other ways to improve traffic flow and to reduce congestion in modern cities. For example, smart cars can inform the intelligent traffic lights (V2I communication) about their approaching. Based on this, traffic lights can attempt to compute an optimal signal plan according to the actual traffic demand. This will be the second layer (vehicle-to-intersection) of the analyzed multi-agent system.

As the third layer, we can assume that the traffic lights also communicate with each other in an attempt to limit the formation of congestion in a wider geographical area. Basically, congestion forms when more vehicles do arrive at

\footnotetext{
${ }^{3}$ Trams and trains can easily be treated as autonomous vehicles on their own. Even pedestrians can be part of this concept, as they can place their demands by pushing a button at intersections, or they can be detected by simple photocells. As they can be informed by traditional lights, theoretically their presence (if orderly) is indifferent to the autonomous vehicles. The difference is simply technical as orderly behaving pedestrians differ from autonomous vehicles only in sensing and signaling.
} 


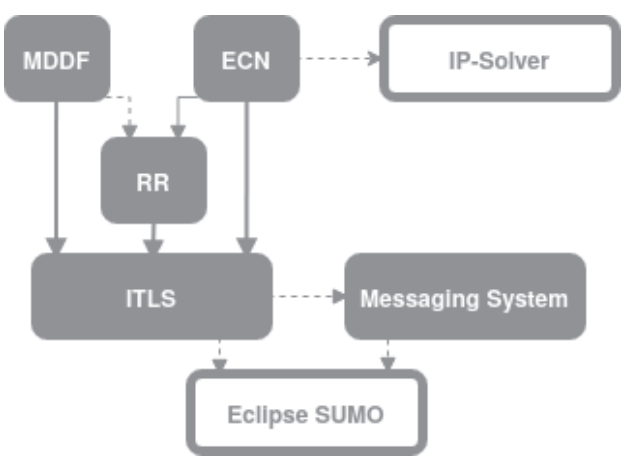

Fig. 2. Functional overview of the proposed system, implemented by extending the Eclipse SUMO microscopic traffic simulator tool. The main functional blocks include a communication module (Messaging System) and the different kinds of ITLS. RR is an ITLS technique on its own, but it is also used by the ECN-method, and contained by the MDDF method as well. ECN method uses an external IP-Solver package.

an intersection than that intersection throughput capacity. If the number of incoming vehicles could be somehow limited, then the congestion could be avoided.

\section{Agent Algorithms And Protocols}

The intelligent multi-agent system delineated in Section III provides a framework in which the actual behavior of particular agents is yet to be defined. In the following, we present some possible algorithms governing the second and the third layer of the system. ${ }^{4}$ We will mainly focus on various algorithms suitable for the intelligent traffic light controllers (ITLS), as these agents participate in both higher system layers.

Considering the second layer, ITLS are only responsible for controlling a single intersection. We will call this an individual scheduling. In the third layer, however, the ITLS share information, therefore we will call it a cooperative scheduling. Actions of a cooperative scheduler control the "lamps" of the scheduler's intersection while these actions are based on both on the scheduler's perceptions and the information received from the topologically neighboring schedulers. For a functional overview, see Figure 2.

\section{A. Simple Individual Scheduling: Round-Robin Protocol}

Individual scheduling, in our solution, is based on wellknown algorithms of scheduling theory. One of the simplest scheduling algorithms is the Round-Robin scheduler (RR). RR provides green-light for every direction periodically. A preemptive version was implemented, which means that greenlight periods can be shorter or even skipped if there are no more smart cars to pass through in a specific direction. In traffic engineering, this is called phase-skipping. In addition to its simplicity, RR provides fair scheduling for all traffic directions.

\footnotetext{
${ }^{4}$ Complex actions in the first layer (i.e. individual vehicles and platoons) also might be defined, but since the time and space is limited for difficult maneuvers in urban scenarios, we do not discuss this possibility here.
}

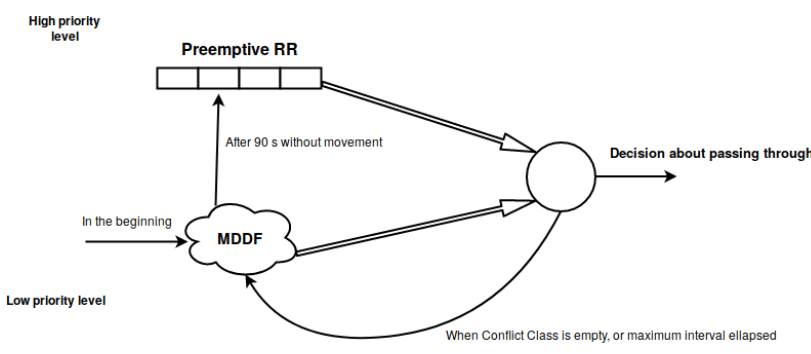

Fig. 3. Functional overview of the MDDF ITLS.

\section{B. Complex Individual Scheduling: $M D D F$}

In principle, there exists an optimal scheduling algorithm, the so-called Shortest Job First scheduler. In the case of urban traffic control, it is a good question what the "shortest job" should mean. One possibility is to pick a vehicle, which is closest to its destination ${ }^{5}$. The idea behind this is that a shorter distance has a smaller impact on the traffic infrastructure, therefore our "job is shorter" too. Let us call it the Minimal Destination Distance First (MDDF) scheduling. However, the MDDF scheduling is not fair in itself. Assume, a lonely car is waiting at an intersection to pass, for example, being at the beginning of a route to a very distant destination. Yet vehicles with significantly closer destinations are continuously arriving. The car with the faraway destination can thus wait forever without getting through this intersection. Assuming that the shortest distances are distributed uniformly between every possible route through an intersection, it is a rare but still problematic case.

If MDDF is combined with RR scheduler [5], [14], then the protocol will be fair. To make it so, the protocol should be a multilayered scheduler with two priority levels. Every direction will be scheduled by the MDDF scheduler when the vehicles arrive at a particular ITLS. If a limited time (here 90 seconds) elapses without receiving a green light for a particular direction, this direction will be scheduled then by an RR scheduler. The RR is at a higher priority, so if there are any directions which must be scheduled by the RR, they will receive green light before those scheduled by the MDDF, see Figure 3 . This way, the scheduler will provide fair scheduling.

\section{Congestion Avoidance in Computer and Road Networks}

The idea of synchronizing signals of neighboring intersections is not a new one. Traffic signal coordination, known mainly as green-waves, has been applied in traffic engineering since 1917, to help the flow of the traffic. Congestion is, however, not a unique phenomenon to the roads of our cities. Computer networking also faces the problem of congestion. If there are more messages to send than the network can handle in a given amount of time, computer networks also become congested.

\footnotetext{
${ }^{5}$ To be precise, the resolution of our scheduling solutions is a so-called conflict-class. The CAVs of a conflict-class can pass through an intersection simultaneously without the risk of an accident. It is analogous to the traditional
} traffic lanes which can receive green lights at the same time. 
Congestion in computer networking can be mitigated in numerous ways. For example, exponential backoff [15] retransmits packets when collisions occur at a point of time, which is selected randomly from an exponentially growing ${ }^{6}$ time range. It is a relatively effective method in computer networks, but it cannot be applied to road traffic.

Another method in computer networking is the sliding window protocol [16]. Its basic idea is that the number of packets transmitted at the same time shall be limited. It would be theoretically applicable to the traffic as well, but in transportation systems, platoons, groups of cars, show many benefits, therefore mitigating or eliminating them might not be so beneficial at the end. Thus, this method is not in the focus of this article.

To the urban traffic we can apply also the Explicit Congestion Notification (ECN) [17] used in the computer networks. The idea behind this algorithm is that the receiver router/intersection informs the sender router/intersection (sends an ECN-signal) when it cannot handle the amount of the incoming messages/vehicles. By catching this notification, the sender is expected to reduce its output until the receiver's further notice.

\section{Cooperative Scheduling: $E C N$}

Implementing the ECN method in the ITLS environment of several intersections is quite a challenging task (see Figure 4). The state-space of such a system can be enormous, therefore storing all the possibilities and searching among them is not necessarily feasible. ${ }^{7}$ However, storing all the possible setups of the "traffic lights" 8 of an intersection is unavoidable if we want to create a pre-programmed ITLS. This ITLS would use this huge list of set-ups in a kind of a look-up-table, therefore given the current state of the traffic and the incoming congestion notifications, the ITLS would be able to select the next signal-phase by searching this particular look-up-table.

Unfortunately, even in modern embedded systems, such look-up-table based solution is almost impossible to implement. Instead of pre-programming the ITLS, signal-phases can be generated in real-time. The calculation of a simple signal phase is mathematically equivalent to solve an integer programming problem (IP). Since modern and powerful IPsolvers are available, this method can be easily ported even to embedded devices.

The variables of the proposed IP are constrained to $\{0,1\}$ values. Every direction will have its corresponding variable, which will be 0 if the direction is to receive a red light, and 1 if it is to receive a green.

The constraints attached to the IP prescribe that only nonconflicting directions can go through the intersection (passing

\footnotetext{
${ }^{6}$ This time range is proportional to the number of the unsuccessful transmissions.

${ }^{7}$ Comparing to traditional phase-skipping, where the number of possible states is linearly proportional to the $N$ number of intersections, here the individual control of directions is necessary. Given $N$ intersections, the number of directions is of order $N^{2}$ (calculated as the maximum number of edges in road network graph).

${ }^{8}$ In an intelligent system, it might be a simple message, not necessarily a physically existing traffic light.
}

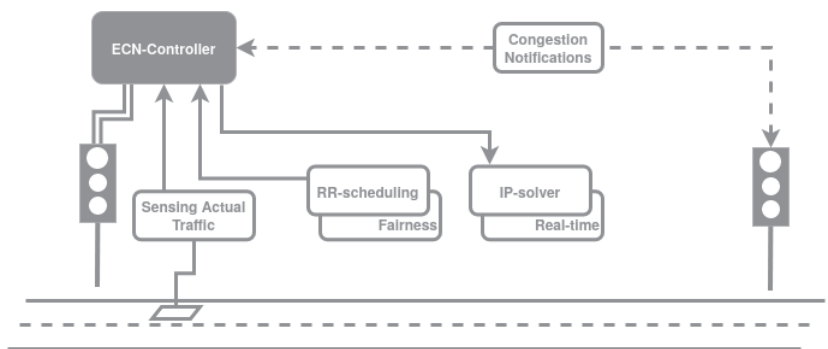

Fig. 4. Overview of an ECN traffic light controller. It is a real-time, fair scheduler. ECN is capable of sensing the actual traffic, and of notifying its neighbors about a forming congestion. When one of its neighbors sends the congestion notification, the ECN-controller will reduce its output accordingly

through the intersection simultaneously without risking collision). Besides, with constraints, some traffic lights can be specifically set to green or to red as desired. The optimum criterion is trivially to maximize the number of directions that currently receive the green light. This approach also avoids creating arterial directions. The lack of an arterial direction, a "main route" might be beneficial in extraordinary situations, because the congested vehicles can reach an alternative route much more easily [5].

When generating a signal phase, at least one direction shall be selected to receive a green light. For this decision, a simple Round-Robin scheduler is used. As it is discussed in Section IV-A, the RR scheduler can provide a fair scheduling $^{\text {}}$. Technically, it means one variable of the IP has to be constrained to 1 , regarding the scheduling decision of the RR.

To make signal plans, individual signal phases have to be calculated periodically. Our solution recalculates signal phases when there are no more cars in the direction which currently receives a green light. A $90 \mathrm{~s}$ time-limit is also set as the maximum time delay between two recalculations. ${ }^{10}$

One problem is yet to solve. It is necessary to decide when congestion is about to form. Without a clear definition of congestion (there is no accepted unequivocal definition of congestion), it is quite a difficult task. Thus based on preliminary simulations, we simply calculated the traffic density, which can provide the highest traffic flow in a given locality. We accept that there is congestion forming when $90 \%$ of this level is reached. The ECN-signal is sent then at this event.

\section{Measurements And Results}

The proposed protocols were tested under a suitably extended version of the Eclipse SUMO, an open-source, microscopic traffic simulation program (see Figure 2). The used network was the BAH-intersection ${ }^{11}$ of Budapest, together with the wider roads of its neighborhood, see Figure 5.

\footnotetext{
${ }^{9} \mathrm{RR}$ scheduler, in this case, is also implemented as a preemptive RR scheduler. This helps increase the traffic flow in the currently popular directions. Therefore it creates arterial directions dynamically, in accordance with the actual traffic demand.

${ }^{10}$ It is alike as in the preemptive Round-Robin scheduler.

${ }^{11}$ Intersection of Hegyalja út, Jagelló út, Villányi út, Budaörsi út and Alkotás utca.
} 


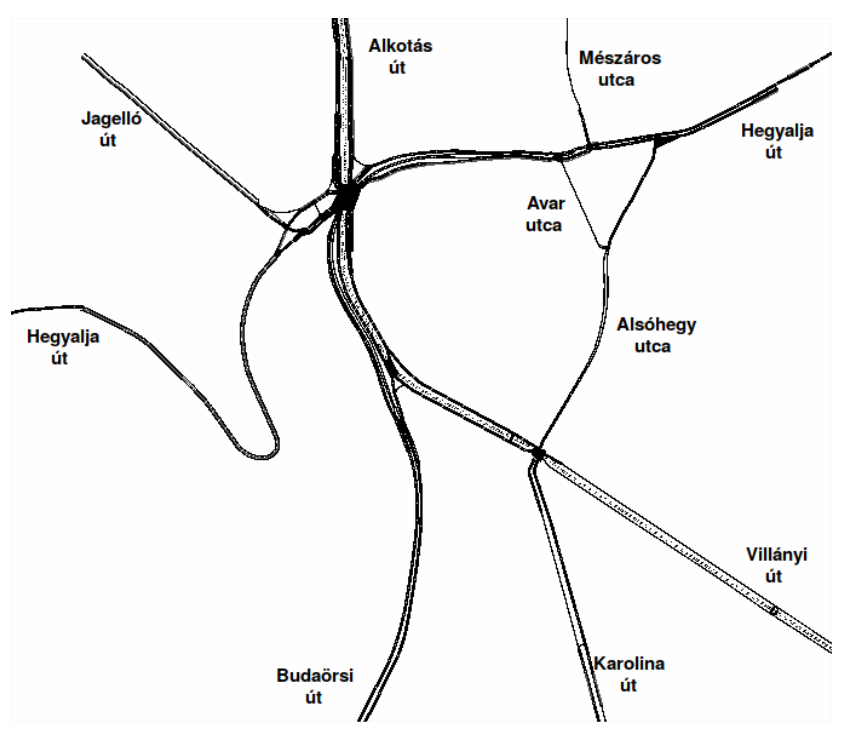

Fig. 5. The simulated network of BAH intersection and its neighborhood.

Regular traffic demands were fed into the simulator (eg. night traffic, morning traffic, noon traffic) as well as some irregular traffic (Budaörsi út is closed) scenarios ${ }^{12}$.

We can see from the results (Table I and Table II), that considering the average waiting time (for example at red lights) and the average traveling time, these indicators are reduced when the proposed intelligent protocols are utilized for irregular traffic situations. On the other hand, in the regular traffic situation, the signal program of the traditional control system (possibly optimized for such regular demands) performs very well (see Figure 6) and the intelligent protocols leave a little margin to the improvement. ${ }^{13}$

TABLE I

Simulation RESUlts OF "IRREgUlar1" CASE

\begin{tabular}{l|rrr} 
Test case & $\begin{array}{r}\text { Arrived } \\
(\boldsymbol{\%})\end{array}$ & $\begin{array}{r}\text { Waiting Time } \\
(\mathbf{s})\end{array}$ & $\begin{array}{r}\text { Average Traveling } \\
\text { Time (s) }\end{array}$ \\
\hline \hline Traditional & 33.81 & 29.68 & 170.55 \\
RR & 29.19 & 12.117 & 174.87 \\
MDDF & 22.77 & 12.41 & 154.02
\end{tabular}

TABLE II

Simulation RESUlTS OF "IRREgUlar2" CASE

\begin{tabular}{l|rrr} 
Test case & $\begin{array}{r}\text { Arrived } \\
(\%)\end{array}$ & $\begin{array}{r}\text { Waiting Time } \\
(\mathbf{( s )}\end{array}$ & $\begin{array}{r}\text { Average Traveling } \\
\text { Time (s) }\end{array}$ \\
\hline \hline Traditional & 38.48 & 36.44 & 199.38 \\
RR & 32.71 & 11.43 & 170.07 \\
MDDF & 34.39 & 10.74 & 176.72
\end{tabular}

The cooperative scheduling was also tried in a regular morning scenario. The BAH-intersection and some of its algorithmically selected neighbors [18] were programmed to

\footnotetext{
${ }^{12}$ Irregularl case. The obstacle is northbound of "Budaörsi út", can be bypassed via Karolina and Villányi streets.

Irregular2 case: Obstacle is southbound of "Budaörsi út", the Obypass route is via "Hegyalja út".

${ }^{13}$ The traffic flow is a commonly calculated value. It is the product of the traffic density $\left(\frac{\text { vehicles }}{k m}\right)$ and the mean velocity of the vehicles $\left(\frac{k m}{h}\right)$. These values can be measured by different types of detectors, cameras, etc.
}

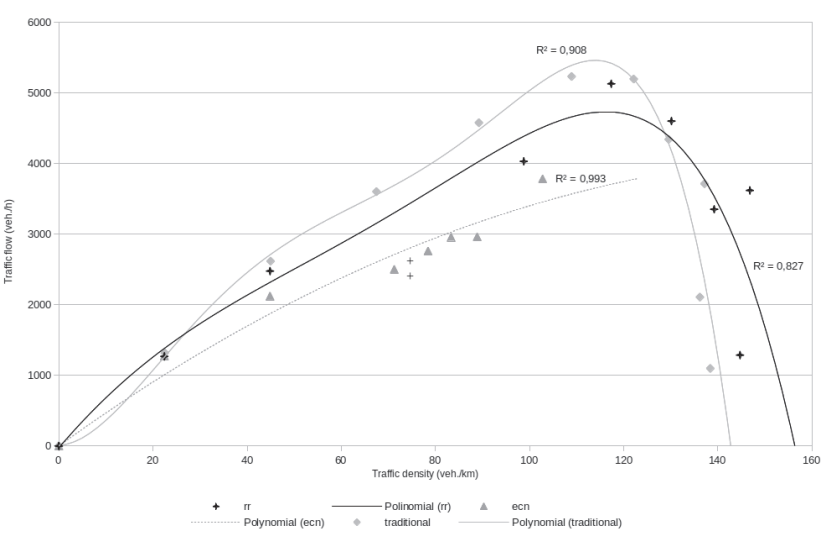

Fig. 6. The results of the simulation, depicted on a Macroscopic Fundamental Diagram of traffic. The values provided by the MDDF ITLS are not shown, because in saturated cases at higher flow of the traffic, they practically coincide with the RR ITLS.

work according to the ECN-algorithm. This algorithm selects a group of neighboring intersections which are coupled to each other. The coupling of the neighboring intersections means a traffic path constraint, i.e. if the vehicles pass one of these intersections, they are constrained to pass the other one also. By running this algorithm, we can identify that the junction of "Jagelló út" with "Hegyalja út", and the junction of "Villányi út" and "Budaörsi út" are coupled to the BAH intersection. Thus, they are governed by the ECN ITLS. Every other ITLS in this system is signaled by simple RR ITLS.

As theoretically expected and visible on the so-called Macroscopic Fundamental Diagram (MFD, see Figure 6), this method limits the flow of the traffic to a certain level, significantly smaller than the maximum achievable flow in this region. Thus, this method cannot be applied to elevate the aggregated number or speed of the vehicles in the simulated scenario. However, in the ECN-coordinated cases, the MFD lacks the descending (jammed traffic) branch of the diagram, therefore the traffic system remains in a stable state.

This proposed method, however, is capable of mitigating congestion in bounded zones of the city traffic [18], especially where the limitation of traffic is desired. Such areas are the residential zones, areas around parks and other recreational facilities, surroundings of hospitals, etc. The major advantage of using an ECN ITLS in such areas, compared to the classical static methods, is that ECN-based control means no inconvenience for the inhabitants and their visitors. On the contrary, commuters cutting-through can be easily banned, as the roads in these zones will not become beneficial alternatives for them.

\section{CONCLUSion}

Representing road traffic as a cooperative intelligent multiagent system provides a framework for modeling intelligent vehicles and infrastructural elements of the cities of the future. To analyze the possible behaviors of the various modeled parts of the road network, the decision making capabilities of the agents have to be defined, best based on the well-tested or mathematically precisely known methods. 
In this paper, we borrowed ideas from computer networks and scheduling theory to create intelligent traffic light controller algorithms. The intelligent single intersection schedulers perform similarly to the traditional control systems in normal traffic conditions. However, in extraordinary situations, intelligent traffic light control can outperform the traditional one. Cooperative scheduling, based on the ECN algorithm and concerting the activity of several intersections, can reduce traffic flow in the whole area. It can be certainly beneficial in some cases, but this method can also avoid reaching the downgrading of the traffic flow.

As the next step of the research, it would be extremely beneficial to investigate whether it would be feasible to set the flow limitation of the ECN-based control to the desired level. If possible, by setting this limit to just below the maximum flow value, we will, therefore, be able to avoid the congestion at a minimal limitation to the maximum achievable flow. Thus, we would be able to keep the traffic flowing close to the theoretical maximum throughput of a given road network.

\section{ACKNOWLEDGMENT}

The research has been supported in part by the BME - Artificial Intelligence FIKP grant of EMMI (BME FIKP-MI/SC) and in part by the European Union, co-financed by the European Social Fund (EFOP-3.6.2-16-2017-00013, Thematic Fundamental Research Collaborations Grounding Innovation in Informatics and Infocommunications). The results presented in the research report were established in the framework of the professional community of Balatonfüred Student Research Group of BME-VIK to promote the economic development of the region. During the development of the achievements, we took into consideration the goals set by the Balatonfüred System Science Innovation Cluster and the plans of the "BME Balatonfüred Knowledge Center", supported by EFOP 4.2.1-16-2017-00021.

\section{REFERENCES}

[1] P. A. Lopez, M. Behrisch, L. Bieker-Walz, J. Erdmann, Y.-P. Flötteröd, R. Hilbrich, L. Lücken, J. Rummel, P. Wagner, and E. Wießner, "Microscopic traffic simulation using sumo," in The 21st IEEE International Conference on Intelligent Transportation Systems. IEEE, 2018, [Online]. Available: https://elib.dlr.de/124092/, DOI: $10.1109 /$ ITSC.2018.8569938.

[2] M. G. Lay, Ways of the World: A History of the World's Roads and of the Vehicles That Used Them. Rutgers University Press, 1992, isbn: 9780813526911.

[3] D. I. Robertson, "Research on the transyt and scoot methods of signal coordination," vol. 56, no. 1, pp. 36-40, 1986, iSSN: 0162-8178.

[4] F. Ahmad, S. A. Mahmud, G. M. Khan, and F. Z. Yousaf, "Shortest remaining processing time based schedulers for reduction of traffic congestion," in 2013 International Conference on Connected Vehicles and Expo (ICCVE), Las Vegas, NV, USA, 2013, pp. 271-276, DoI: 10.1109/ICCVE.2013.6799805.

[5] L. Alekszejenkó and T. Dobrowiecki, "Intelligent vehicles in urban traffic - communication based cooperation," The IEEE 17th World Symp. on Applied Machine Intell. and Inform. (SAMI 2019), Herl'any, Slovakia, Jan 2019, DoI: 10.1109/SAMI.2019.8782778.

[6] K. Dresner and P. Stone, "A multiagent approach to autonomous intersection management," Journal of Artificial Intelligence Research, vol. 31, pp. 591-653, 2008, Dor: 10.1613/jair.2502.

[7] S. Nigarnajagoool and H. Dia, "A multi-agent approach to real-time traffic signal optimisation," in 29th Australasian Transport Reserach Forum, Surfers Paradise, Queensland, Australia, September 2006, isbn: 1877040568 .
[8] M. Girianna and R. Benekohal, "Dynamic signal coordination for networks with oversaturated intersections," Transportation Research Record, vol. 1811, pp. 122-130, 01 2002, Dor: 10.3141/1811-15.

[9] A. Deligkas, E. Karpas, R. Lavi, and R. Smorodinsky, "Traffic light scheduling, value of time, and incentives," in Proc. of the TwentySeventh International Joint Conference on Artificial Intelligence Main track., July 2018, pp. 4743-4749, DoI: 10.24963/ijcai.2018/659.

[10] P. Wagner, R. Alms, J. Erdmann, and Y.-P. Flötteröd, "Remarks on traffic signal coordination," in EPiC Series in Computing, vol. 62, 08 2019, pp. 244-255, DoI: 10.29007/flbm.

[11] A. Bazzan, "A distributed approach for coordination of traffic signal agents," vol. 10, pp. 131-164, 2005,

DOI: $10.1007 / \mathrm{s} 10458-004-6975-9$

[12] L. Kuyer, S. Whiteson, B. Bakker, and N. Vlassis, "Multiagent reinforcement learning for urban traffic control using coordination graphs," in Machine Learning and Knowledge Discovery in Databases, W. Daelemans, B. Goethals, and K. Morik, Eds. Berlin, Heidelberg: Springer Berlin Heidelberg, 2008, pp. 656-671, DOI: 10.1007/978-3-540-87479-9_61.

[13] B. Beak, "Systematic analysis and integrated optimization of traffic signal control systems in a connected vehicle environment," 2017. [Online]. Available: http://hdl.handle.net/10150/626304

[14] L. Alekszejenkó and T. P. Dobrowiecki, "Sumo based platform for cooperative intelligent automotive agents," in SUMO User Conference 2019, ser. EPiC Series in Computing, M. Weber, L. Bieker-Walz, R. Hilbrich, and M. Behrisch, Eds., vol. 62. EasyChair, 2019, pp. 107123, [Online]. Available: https://easychair.org/publications/paper/ Vp8w, DoI: $10.29007 / \mathrm{sc} 13$

[15] "Ieee standard for ethernet," IEEE Std 802.3-2015 (Revision of IEEE Std 802.3-2012), pp. 1-4017, 2016, isbn: 978-0-7381-9626-8.

[16] V. Jacobson, R. Braden, and D. Borman, "Tcp extensions for high performance, rfc 1323." The Internet Society, Network Working Group, May 1992, [Online]. Available: https://www.ietf.org/rfc/ rfc1323.txt.

[17] S. Floyd, K. K. Ramakrishnan, and D. L. Black, "The addition of explicit congestion notification (ecn) to ip, rfc 3168." The Internet Society, Network Working Group, September 2001, [Online]. Available: https://tools.ietf.org/html/rfc3168.

[18] L. Alekszejenkó and T. P. Dobrowiecki, "Alleviating congestion in restricted urban areas with cooperative intersection management," in IntelliSys 2020, September 2020, (Accepted for publication in Springer series "Advances in Intelligent Systems and Computing").

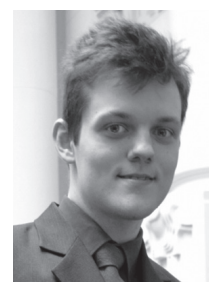

Levente Alekszejenkó was born in Fehérgyarmat, Hungary, in 1996. He is a member of the Balatonfüred Student Research Group. He received the B.Sc. degree in computer science engineering from Budapest University of Technology and Economics, Budapest, Hungary in 2019. His M.Sc. studies in computer science engineering are currently ongoing at the Budapest University of Technology and Economics, Budapest, Hungary. He is currently working at evopro Innovation Ltd., Budapest, Hungary as a Junior Software Engineer. His research interests include multi-agent intelligent systems, especially their application in intelligent transportation systems and road traffic simulation.

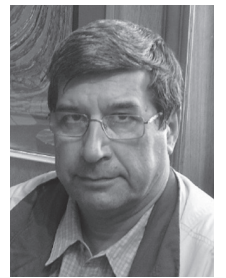

Tadeusz P. Dobrowiecki was born in Warsaw, Poland, in 1952. He received the M.Sc. degree in electrical engineering from the Technical University of Budapest, Budapest, Hungary, in 1975, the Ph.D. (candidate of sciences) degree in 1981, and the DSc (Doctor of Sciences) degree in 2018 , both from the Hungarian Academy of Sciences.

After spending one year as a Professional System Engineer, he joined the Department of Measurement and Information Systems, Budapest University of

Technology and Economics, Budapest, Hungary, as a Staff Member, where he is currently a Full Professor. He is involved in teaching artificial intelligence and system identification. His current research interests include technical applications of artificial intelligence, and advanced system identification problems. 